\title{
DESIGN MODELLING AND ANALYSIS OF STANDARDIZED HYDRAULIC POWER PACK
}

\author{
Srinivas $\mathbf{R}^{1}$,Mrs. Manjula ${ }^{2}$, Saira Das $^{3}$, Bharadwaj $^{4}$ \\ Isrinivasr038@gmail.com
}

\begin{abstract}
A hydraulic system is a group of hydraulic elements arranged in an order and using these hydraulic elements power is transmitted using a confined liquid i.e., oil. Generally hydraulics will be used where force required is more to do the function. Hydraulic power units are drive system for hydraulic machines. A hydraulic assembly is intended exclusively for integration into machines or systems or to be assembled with other components to form a machines or systems. The product may only be commissioned if it has be integrated into the machine or systems for which it is designed. Hydraulic power units are drive systems for hydraulics machines.
\end{abstract}

Keywords: Hydraulic System, Hydraulic Power Pack, Catiav5, Ansys12, Directional Valve.

\section{INTRODUCTION}

A Hydraulic system is defined as the study of fluids at restsor in motions with respect to engineering applications. A fluid may be either of water, oil etc. $\square$. [1].

Magnitude of force transferred is directly proportional tothe surface area $(\mathrm{F}=\mathrm{P} * \mathrm{~A}) \cdot[2]$

Pressure $=$ Force/Area [3]

\section{OBJECTIVES}

Project aims at developing standardized elements tool to prepare hydraulic circuit for variety functions.

[1] Clamping.

[2] Sequencing.

[3] Pressing.

[4] Testing.
[5] Feedcircuits.

[6] Counter Balance circuit.

[7] Proportional circuit.

[8] Servocircuit with minimal times.

\section{ELEMENTS OF HYDRAULIC SYSTEM}

- Pumps,

- Actuators

- Rotary-(motors )

- Control Elements- Valves :Pressure Flow and Directional

- Accessories - Reservoirs, coolers, filters,storage units tanks

- Accumulators

- Pipes Fittings \& Hoses

Pressure/ Flow Feedback devices

Figure1: Proposed machine layout

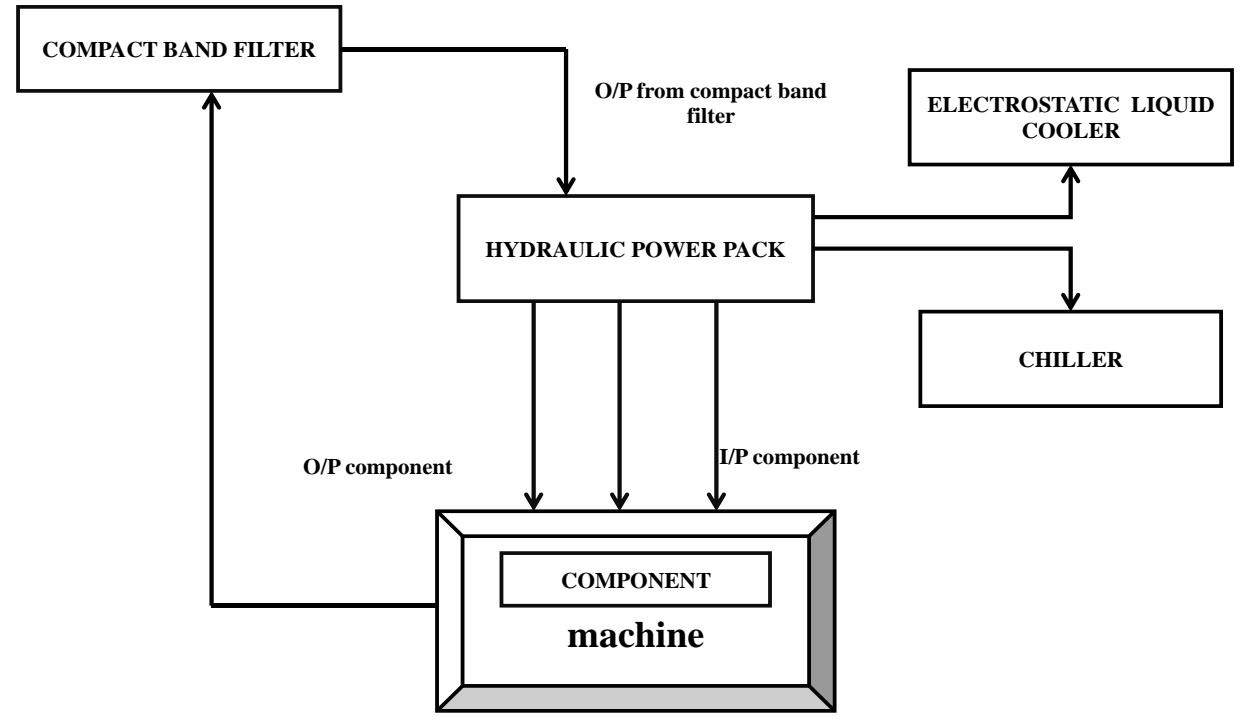




\section{METHODOLOGY}

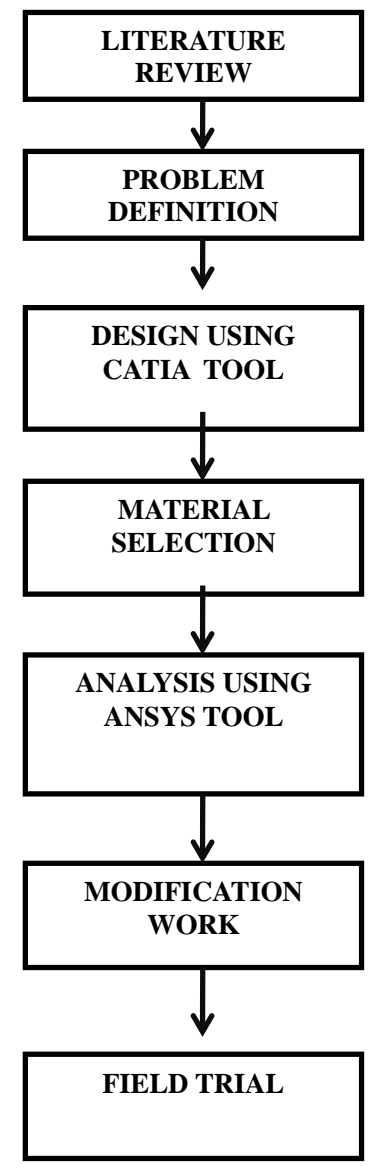

\section{FLOWCHART OF FINITE ELEMENT ANALYSIS}

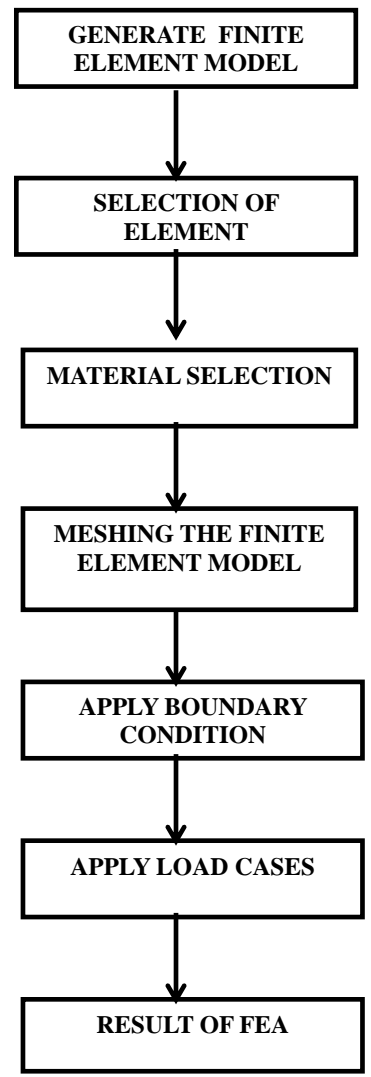

\section{DESIGN OF EXPERIMENT}

The below sketch shows three dimension of standardized hydraulic power pack consists of different parts and this was modelled and designed by using famous software known as catia v5.

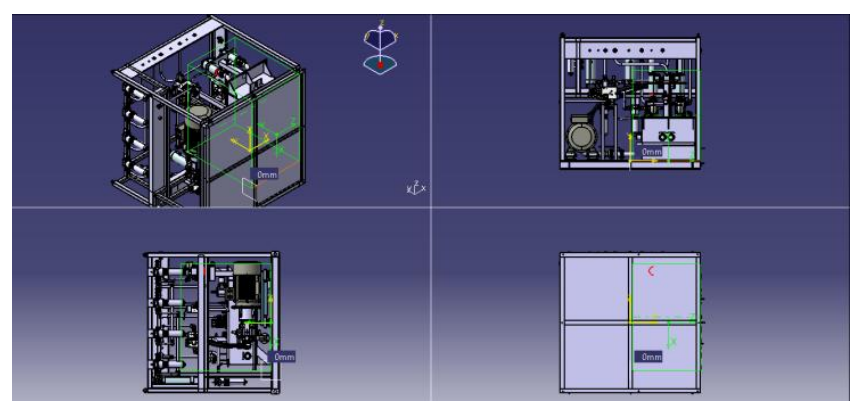

\section{RESULTS}

Tank Analysis

Model:

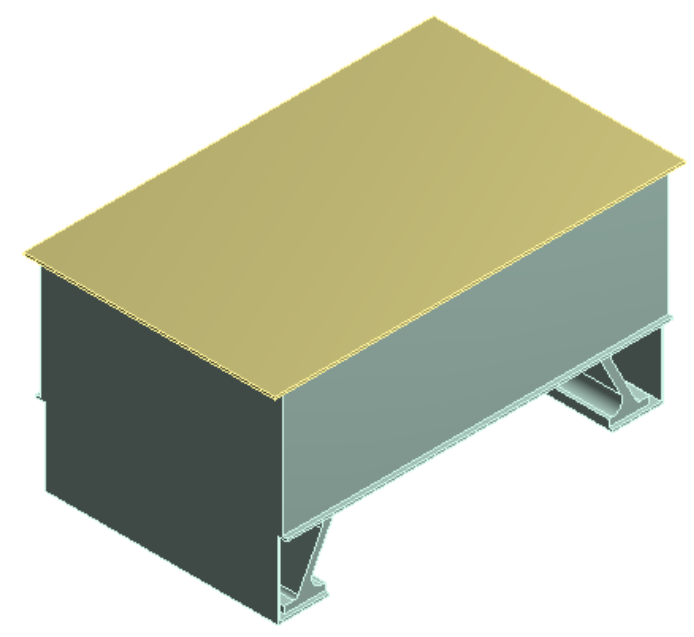

\section{BOUNDARY CONDITIONS}

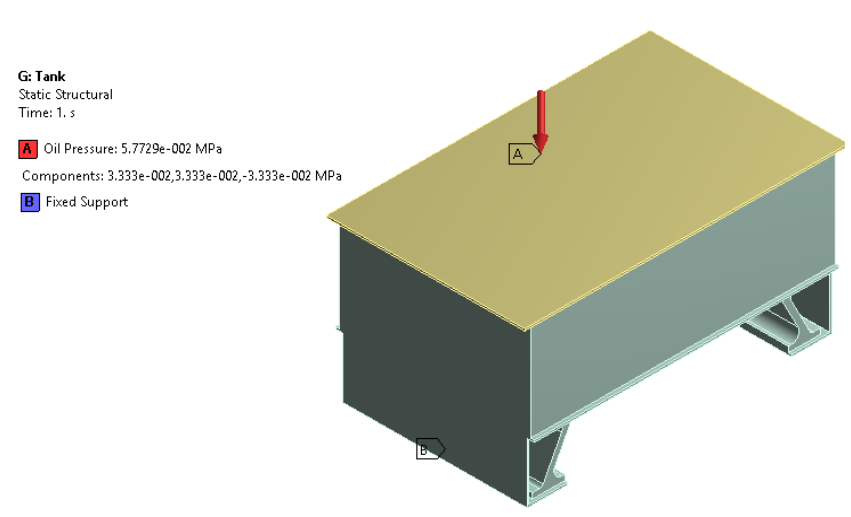

TANK CALCULATION

- Mass $=630$ liters

- Volume $=1817 * 1019 * 758$ 


\section{FILTER ANALYSYS}

\section{Geometric Model}

\section{Before modification}

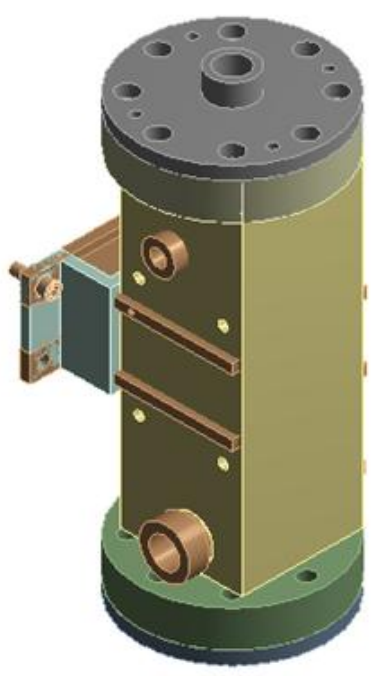

\section{After Modification}

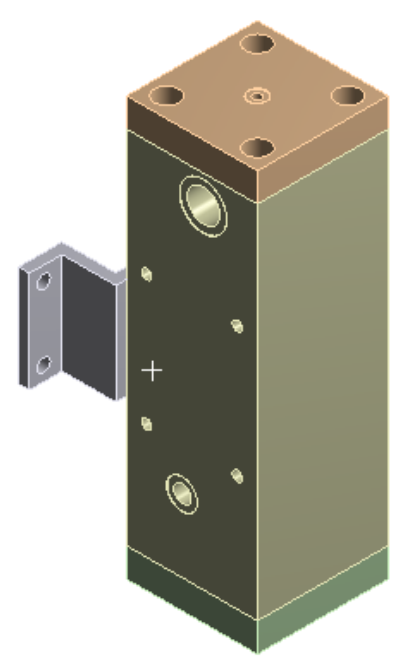

\section{BOUNDARY CONDITIONS}
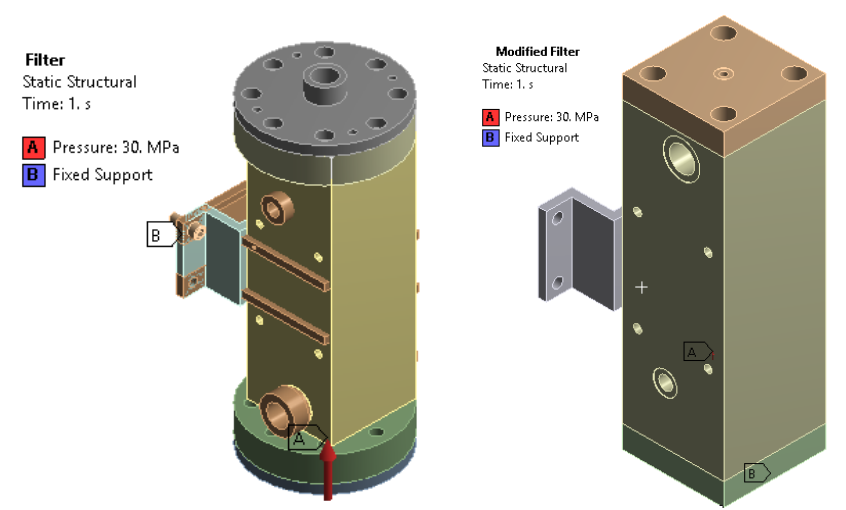

\section{MATERIAL PROPERTY OF FILTER}

- Element

- Filter Pressure

- Velocity

- Temperature

- Mass Flow Rate

- Young'ssModulus

- Poisson's Ratio

- Ultimate Stress

$\begin{array}{ll}= & \text { Filter(Mild Steel) } \\ = & 300 \mathrm{bar} \\ = & 6-7 \mathrm{~m} / \mathrm{s} \\ = & 50 \mathrm{deg} \text { Celsius } \\ = & 10-100 \mathrm{lpm} \\ = & 210 \mathrm{GPA} \\ = & 0.3 \\ = & 500 \mathrm{MPA}\end{array}$

\section{BENEFITS}

- Cost minimization.

- Symbol library with part no of different suppliers i.e Bocsh-Rexroth, yuken, Nachi, Voith.

- Function block library with parts list.

- Circuit design for the hydraulic circuit to be carried out with the help of created libraries \& tool during the project.

\section{CONCLUSIONS}

- Detaileddesignis done as per customerrequirements.

- Required design modifications.

- Results obtained with design calculations using FEM approach which is carried out in ANSYS.

\section{REFERENCES}

[1].Wang Jing, Gong Guofang, Yang Huu "Improvinggdynamicsquality of hydraulic system withs flows" DSCC2008-2103, October 20-22, 2009, 2009 ASME.

[2].Wenyoung Ma, Delun Wong*, Kwun-Lon Ting, "Automated conceptual design of hydraulic system" IDETC/CIE 2010, August 3-6, 2010 ASME.

[3].CarlA.Nelson,AbolfazlPourghodrat,"Energy harvesting from

verticaldeflectionsofrailroadtrackusingHydraulicsystemforsi mproving railroad tracksafety" IMECE2013-542102, November 11-17, 2013 ASME.

[4].Zengmeng Zhang ,

"Proportional pressure control of hydraulic system for water

Hua Zhou, water injection moulding modelling simulations"DETC2014-24256,NOVEMBER16-18ASME [5].Aditya Shah,Christiaan J.J. Paredis, "Combining mathematical programming and syste for automated component sizing of Hydraulic systems" DETC2012-22580, August 16-17, 2014ASME.

[6].Amand M. Plagge, Lee Jestings, Brenden P. Epps "Nexttgeneration hydrokinetic powerstake-off viaa novel variable stroke hydraulic system" MAE2014-24095, June 813, 2014 ASME.

[7].Milos Vukovic, Sebastian Sgro, Hubertus Murrenhoff, "Steam A mobile Hydraulicsystem with engineering integration" FPMC2014-4408, October 6-9, 2014 ASME.

[8].Cristian Cardoso Locateli, Paulo Leonel Teixeira, "Digital Hydraulic system " FPNI2014-7839, June 11-13, 2014". 